\title{
Interventional cardiology during the COVID-19 epidemic
}

\section{Hellmuth Weich*, Jens H itzeroth", Sajidah Khan ${ }^{\dagger}$, David Kettles ${ }^{\ddagger}$, Ahmed Vachiat ${ }^{\dagger}$ and M piko $\mathrm{N}$ tsekhe\#}

\begin{abstract}
"Division of Cardiology, Department of Medicine, Stellenbosch University and Tygerberg Hospital, Bellville, South Africa "Division of Cardiology, Department of Medicine, Faculty of Health Sciences, University of Cape Town and Groote Schuur Hospital, Observatory, Cape Town, South Africa

tBusamed Gateway Hospital, Umhlanga, Durban, South Africa ¥St Dominics Private Hospital, and Frere Hospital, Southernwood, East London, South Africa

Milpark Hospital,Wits Donald Gordon Medical Centre, Johannesburg, South Africa
\end{abstract}

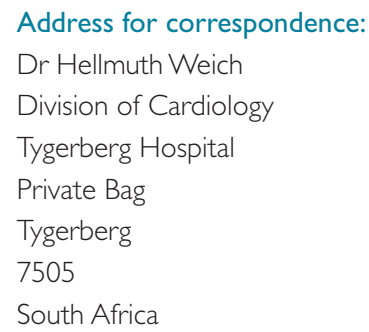

\section{INTRODUCTION}

Since the outbreak of the COVID-19 pandemic towards the end of 2019, it has spread rapidly throughout the world and healthcare has had to adapt to the problem in ways that were previously thought unimaginable and certainly not anticipated. In a short space of time, a huge body of evidence has been gathered and disseminated, but it remains difficult to distinguish between hype, panic and fact. Specifically, it has been difficult to find facts that help interventional cardiologists adapt their clinical practice and patient care algorithms in a safe manner that benefits short- and long-term outcomes. However, the one truth that has emerged is that systems that are better prepared tend to do better than those that are not. To this end, we aim to provide some direction on how interventional cardiology may be affected and should be managed during the pandemic.

The unique and specific challenge that COVID-19 brings to the interventional cardiologist is that we are no longer considering only what is best for the patient, but also the risk to healthcare

\section{ABSTRACT}

The impact of the COVID-19 pandemic on our lives is unprecedented and major adjustments to our practices as physicians are required. Although our comments are applicable at the time of writing, the situation changes daily and the content of this article should be adjusted accordingly.

Cath lab: An unambiguous cath lab protocol should be drawn up for each facility, appropriate to local circumstances. This should include standard procedures in preparation for arrival at the lab, in the performance of procedures, and, importantly, in maintaining due diligence when removing protective gear. All team members should be well trained in these procedures. Acute coronary syndromes: Standard timing for the invasive management of patients should not change during the pandemic. Due to delays often unavoidable during the pandemic, alternative strategies such as thrombolysis may be more readily available and therefore more appropriate.

Drugs: The sick COVID-19 patient often represents a pro-thrombotic state and operators should ensure adequate anti-thrombotic therapy. Knowledge of interactions between cardiac drugs and investigational antiviral treatments is important.

Elective procedures: Patients with chronic cardiac conditions are at high risk and may require non-urgent procedures to avert major complications. Selecting these cases requires consideration of multiple risks and benefits. SAHeart 2020; 17:296-304

practitioners, other patients, and the healthcare system. Although this review will be applicable at the time of writing, it is important to adjust the management of cases to the changes in the epidemic and healthcare capacity.

\section{RISK TO HEALTHCARE PROVIDERS}

The risk of healthcare providers (HCP) contracting COVID- 19 in a specific setting is difficult to judge, because of different levels of protection and exposure in different environments. In a large cohort studied by the US Centres for Disease Control and Prevention, occupational status was known for 49370 out of 315531 COVID-19 cases and 9282 (19\%) of these were HCPs. They reported contact with confirmed COVID- 19 cases in a healthcare facility in only $55 \%$ of cases. 
Only 10\% were hospitalised, 5\% were admitted to ICU, and mortality was $0.6 \%$.(1)

A recent systematic review of 64 publications on the epidemiology and risk to HCPs concluded that HCPs were at high risk for infection by virtue of significant exposure in the workplace, but that the use of appropriate protective measures and equipment reduced this risk considerably. ${ }^{(2)}$ When managing individual patients, the risk to healthcare providers depends on the risk of the patient having COVID-19 and the risk of the specific procedure.

\section{Patient risk categories}

The WHO have provided definitions to classify patients based on the access to test results and patient symptoms, which give guidance to HCP of their risk exposure (see Table I). ${ }^{(3)}$

\section{Procedure risk categories}

The ESC have provided some guidance in classifying procedure risk and therefore recommendations for the level of personal protective equipment (PPE) required. Local availability of level III protection (the highest, requiring full-face respiratory protective devices or powered air-purifying respirators) is extremely limited and probably not applicable. For the interventional cardiologist, this implies that the highest level of protection is level II, which includes:

Disposable surgical cap
Medical protection mask (N95/ FFP2)
Work uniform
Lead apron
Gown
Goggles/visor

Disposable surgical gloves (ideally 2 pairs to enable safe removal) $)^{(4)}$

The above is considered appropriate for the following procedures on patients classified as suspected/probable or confirmed:

- Trans-oesophageal echocardiogram (TOE)

- Coronary angiography and PCl

- EP studies

- Cleaning of instruments used (e.g. TOE probes, stethoscope) $)^{(5)}$

\section{Patient COVID-19 status}

If a widely available, cheap test with immediate results were available, it would make sense to test all patients prior to admission. This would make decision-making in the hospital vastly simpler, despite the fact that a negative test does not exclude the disease. Availability of tests is however variable and results may take between 4 hours and 7 days. An individualised approach is therefore required depending on local circumstances, although the ideal should be to test all patients on or prior to admission. ${ }^{(6)}$ Where such testing is not available, all patients coming to the lab should wear surgical face masks. Patients who are intubated or presenting with STEMI or very high risk NSTEMI requiring urgent angiography, should be assumed to be COVID-19 positive. ${ }^{(6)}$ We, however, have to concede that this has different implications for different environments. Options range from standard PPE for most cases, with an upgrade to full level II PPE in the case of a resuscitation in some hospitals, to full donning and doffing of level II PPE for all cath lab staff for all procedures, and cleaning of the lab after each such case in others.

\section{TABLE I: Patient risk status as defined by the $\mathrm{WHO}^{(3)}$}

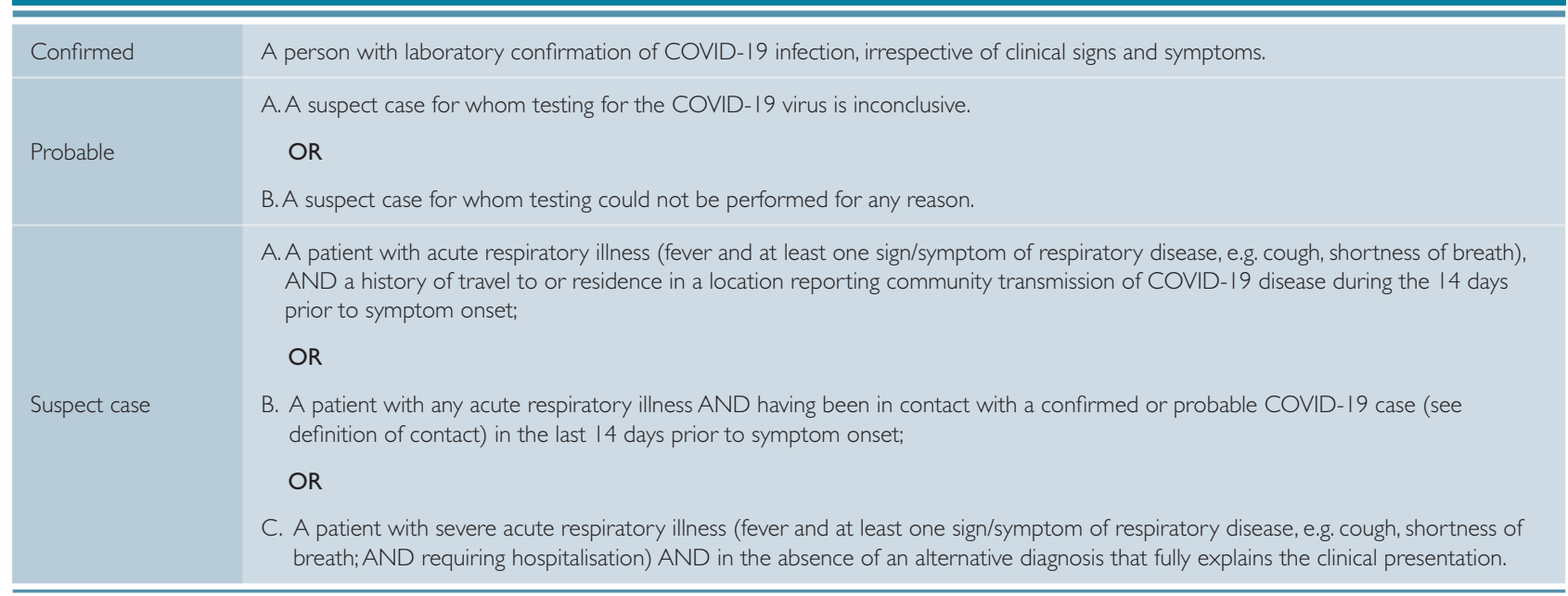




\section{CATH LAB PREPARATION}

It is recommended that hospitals have standard operating procedures in place for COVID-19 positive/suspected patients and that these are well communicated to all personnel. If a hospital has more than one cath lab, then a single lab should be allocated to performing all procedures on COVID-19 positive patients. When choosing a lab dedicated to COVID- 19 patients, one should bear in mind that the ESC recommends that the air-conditioning in the cath lab should do a minimum of 15 air replacements/hour, but ideally 30.(5) The dedicated lab should have all the equipment required outside the lab (with a designated runner to bring items to the door) or stored in cupboards with closed doors. Most cases performed will remain on COVID-19 negative patients, but having the lab set up in this manner will greatly reduce the time required to clean up after cases involving COVID-19 positive patients.

\section{Donning and doffing}

All HCPs involved should be trained in the correct use of PPE. These procedures should be done under supervision and especially doffing should always be supervised by a trained person - the so called "Dofficer". (4)

\section{Prior to patient's arrival}

- Coordinate transport to ensure the patient does not have to wait outside the lab

- For unstable patients: consider elective intubation under controlled circumstances prior to the procedure

Ensure the minimum number of people are in the lab

\section{The procedure}

- Only people involved in the procedure should be in the room and nobody should leave the room until completion

- Resuscitation is extremely high risk for aerosolisation of the virus and each lab should have a clear standard operating procedure for this

\section{After the procedure}

This part is probably the most important safety aspect of dealing with COVID patients. The EAPCI position statement recommends the following:

Keep the door closed

- Supervised doffing. This should be done inside the lab. The facial respirator should be removed outside the cath lab at the end

Do not squeeze contaminated materials into the waste container in order to avoid aerosol generation

- Personnel to leave the room

- Personnel should be monitored for symptoms of COVID on a regular basis. ${ }^{(6)}$

\section{ACUTE CORONARY SYNDROMES}

The phenomenon of decreased referrals of acute coronary syndrome (ACS) has been identified internationally during the COVID-19 pandemic and is likely to be of multifactorial origin. ${ }^{(7)}$ Possible causes include a reduction in stressors such as traffic, pollution and smoking, or fear of presenting to potentially contaminated hospital environments. The cardiovascular presentations of ACS patients with COVID- 19 can be complex, with varying presentations of STEMI, stress cardiomyopathy, non-ischaemic cardiomyopathy, coronary spasm or non-specific myocardial injury. $(8,9)$

These considerations become more critical where staffing reserves are marginal, and where many South African hospitals are delivering $\mathrm{PCl}$ with a single catheterisation laboratory within a hospital facility. Careful evaluation is needed: which aspects of contemporary care are likely to bring greatest benefit to the patient and community, and which aspects are most likely to jeopardise the ongoing service delivery expected of our interventional units?

\section{STEMI}

The global experience of managing STEMI during this pandemic has been encapsulated as "large delays in the small number of patients with STEMI seeking medical help".(10) These delays are multi-level, inter-alia beginning with a reluctance of patients to come to the hospital, continuing to delays in diagnosis in the emergency room where triage is slowed by screening measures, and further exacerbated by time-consuming laboratory preparation measures, including the donning of PPE. These delays occur in a context where the well-established "time is muscle" paradigm demands very rapid decision-making and delivery of reperfusion therapy.

\section{Differential diagnosis}

COVID-19, similar to other viral syndromes, may mimic a number of other conditions and each patient therefore requires a quick evaluation to decide if the presenting complaint is likely to be a primary coronary problem that may be improved by early invasive management. In one study, $40 \%$ of COVID-19 patients with chest pain and ST elevation had no evidence of obstructive coronary disease at angiography. (I) Alternative diagnoses include myocarditis, Takotsubo cardiomyopathy and shock due to severe COVID-19 disease. ${ }^{(12,6)}$ We may additionally expect increased susceptibility of chronic coronary artery disease patients to an acute myocardial event should they become COVID-19 infected - a phenomenon well described in relation to the influenza virus. ${ }^{(13)}$ There is also evidence to suggest that patients with COVID-19 are more prone to thromboembolic events and pulmonary embolism must remain in the differential diagnosis. ${ }^{(14)}$ 
Timing and reperfusion strategy

The COVID-19 pandemic should not compromise early reperfusion in all eligible patients (symptoms of ischaemia of $<12$ hours duration and persistent ST-segment elevation in at least two contiguous ECG leads. ${ }^{(15)}$ In contemporary South African practice, relatively few patients usually have access to primary $\mathrm{PCl}$ and we are accustomed to fibrinolysis as first-line STEMI reperfusion therapy. For most South African hospitals this means that current reperfusion protocols remain applicable, though we may need to increase our flexibility as to where such treatments can be administered.

The maximum time delay for primary $\mathrm{PCl}$ should remain 120 minutes from first medical contact. Consideration should be given to a lysis first strategy, even in units traditionally pursuing primary $\mathrm{PCl}$. This is likely to expose the least number of HCP to potential risk. Furthermore, where rapid testing is available patients can await test results, while effective reperfusion therapy has already been administered. In our context an estimated 60 minutes of extra delay in time to $\mathrm{PCl}$ (related to COVID-19-specific hospital adaptations) will likely delay many patients beyond the acceptable 120 minutes of $\mathrm{PCl}$-related reperfusion delay.

It seems probable then that immediate triage to primary $\mathrm{PCl}$ will frequently require extra delays and costs to the system, as outlined in the Cath lab Preparation section. These obstacles may become prohibitive in centres without the luxury of a COVID- 19 dedicated lab.

\section{Pharmaco-invasive approach}

Usually, guideline mandated pharmaco-invasive practice is to triage STEMI patients to the nearest $\mathrm{PCl}$-capable hospital, after administration of thrombolytic reperfusion therapy at the peripheral hospital. The need for delivery of reperfusion therapy at the primary hospital must be highlighted at this time COVID-19 brings this opportunity to re-evaluate our usual practices. STREAM-like protocols have, even pre-COVID-19, recommended that timeous fibrinolytic therapy should be given at first suitable point of medical contact. ${ }^{(16)}$ Under pandemic conditions, transfer to a $\mathrm{PCl}$-capable centre is a contentious issue and dependant on:

\section{Availability of COVID- 19 testing}

Availability of early COVID-19 test results

Result of the thrombolytic therapy

Likely benefit of the intervention, considering the extent of myocardium at risk and time course of the infarct

These factors will differ between healthcare systems and need to be individualised, although the aim should still be to consider every patient for angiography within a reasonable time frame.
Known COVID-19 negative status may expedite transfer arrangements and will certainly facilitate best use of facilities at the receiving hospital.

\section{Failed lysis}

There will be a significant group of STEMI patients in whom either fibrinolysis is contraindicated, or where it has failed to restore perfusion. This group is not insignificant: Post fibrinolysis, just over $50 \%$ of patients may require such rescue procedures. ${ }^{(17)}$ Such patients, regardless of COVID-19 status, will require consideration for rescue $\mathrm{PCl}$.

\section{COVID-19 complications}

Where severe COVID- 19 complications have limited prognosis more than the risk attributable to the infarct, it may be considered futile to proceed with angiography. Some virus complications may contra-indicate the angiogram (severe renal dysfunction). We consider that a multidisciplinary team caring for the patient could assist in identifying such cases of likely medical futility, which may then be appropriately considered for compassionate conservative medical therapy. ${ }^{(8)}$

\section{Multivessel disease}

When a patient has non-culprit lesions that need intervention, operators should consider performing these at the same session to minimise the number of cath lab visits.

This complex weighing of risks will have to be patient-specific, with a clear understanding that we are operating in an area where we have little data.

It must be clearly understood that supervision and leadership is important, and we must strive to spare overburdened caregivers with additional stressors in an already potentially damaging environment. Unit-specific treatment protocols can be constantly updated to reflect unique circumstances, and innovative use of new audio-visual networking technology can allow multidisciplinary team decisions to be agreed on - even in acute, complex revascularisation cases. Such sharing of decision-making may be anticipated to be beneficial to the attending physician, other staff, and certainly the patient.

\section{NSTE-ACS (NON ST ELEVATION ACUTE CORONARY SYNDROMES)}

The initial triage of patients presenting with chest pain syndromes with ECG changes and/or elevated troponins should include consideration of other diagnostic possibilities, similar to those discussed under STEMI (above).

As with STEMI, and for similar reasons, all patients presenting with NSTE-ACS should be screened for SARS-CoV2 at first medical contact, or as soon as possible thereafter. 
Conventional management algorithms for NSTE-ACS identify categories of risk which define the likelihood of ensuing major adverse events, and the need for early invasive management strategies.(18) During this pandemic, accurate identification of the high risk patient becomes a critical step that will allow unusually limited resources to be accurately focused, and prevent low risk patients being exposed unnecessarily to hospital risks.

\section{Very high risk}

The recently published EAPCI position statement summarises well known, very high risk features: "haemodynamic instability or cardiogenic shock, recurrent/ongoing chest pain refractory to medical therapy, life-threatening arrhythmias or cardiac arrest, mechanical complications of $\mathrm{MI}$, acute heart failure, and recurrent intermittent ST-elevation". (6) This cohort of patients should be managed with the same degree of urgency as the STEMI group and therefore also be presumed to be COVID- 19 positive until proven otherwise. If the medical facility of first contact cannot deliver angiography under these particular circumstances, such very high risk patients will require immediate transfer to an appropriate COVID-19-lab-equipped facility. Angiography should not be delayed. ${ }^{(5)}$

\section{High risk}

High risk patients include those that are troponin positive with dynamic ST segment changes, high Grace or TIMI scores or ongoing symptoms. If they can be stabilised for 24 hours until a virus test result is available, their ongoing management is much simplified, and they follow usual protocols. ${ }^{(6)}$ Although there is benefit for early (within 24 hours) interventional procedures, it may often be reasonable to extend that 24 hour period until the result of a COVID- 19 screening test is available. Clinical evaluation of the patient's progress will dictate the permissible additional delay.

\section{Intermediate risk}

Intermediate risk patients should be stabilised with optimal medical therapy, which may allow sufficient time to obtain virology results. This category includes patients with established NSTEMI based on cardiac troponins AND at least one of the following: diabetes mellitus, renal insufficiency, LVEF $<40 \%$ or congestive heart failure, early post-infarction angina or prior $\mathrm{PCl} / \mathrm{CABG}$. Where coronary CT is available with appropriate experience and expertise in interpretation, these patients may be candidates for non-invasive evaluation to exclude high-risk anatomical features which may require invasive angiography such as left mainstem disease. ${ }^{(5)}$ While this may reduce exposure of cath lab staff to COVID-19, this may be offset by the exposure to the CT angiography staff.

\section{Low risk}

Low risk patients should be discharged as soon as stabilised and followed with non-invasive ischaemia testing.

\section{DRUGS IN THE CATH LAB}

The use of investigational therapies in COVID-positive patients may influence drug selection and dosages in the cath lab. During the pandemic, the majority of patients taken to the lab will comprise high-risk ACS patients. ACS is a recognised prothrombotic state and as we learn more about the COVID-19 virus, it is being increasingly associated with a systemic coagulopathy, and anticoagulation is linked to better survival among patients hospitalised with the condition. ${ }^{(14)}$ Drug therapy will focus on adjustments to standard practice that need to be considered in the cath lab and will note the potential interactions with current antiviral therapies.

\section{Conscious Sedation}

The usual pharmacological agents such as midazolam or diazepam are adequate, unless standard doses are exceeded, when respiratory depression becomes a concern. If opioids such as morphine or fentanyl are required for additional pain control, delayed gastric emptying or vomiting induced by these agents may impact on drug absorption. As anti-platelet P2Y I2 inhibitor loading for ACS often occurs in the lab at the time of intervention, those given opioids in a 2-hour span before or after the procedure may require bridging with a parenteral agent. ${ }^{(19)}$ Glycoprotein Ilb/llla inhibitors may need to be initiated in the lab if the patient was not on chronic P2Y I 2 inhibitor therapy or insufficiently pre-treated with antiplatelet therapy prior to arrival in the cath lab.

\section{Anti-thrombotic treatment}

The coagulation abnormalities observed in those with more severe COVID-19 infections, which include elevated D-dimer levels, mild thrombocytopenia, a prolongation of prothrombin and thrombin times, and disseminated intravascular coagulation, as well as therapies administered prior to arrival in the cath lab, may impact on the selection and dosages used in our standard protocols. Listed below are the recommended regimens for patients undergoing primary $\mathrm{PCl}$ (Table II) for STEMI. The following medications may need adjustments if patients are on concomitant anti-viral therapies.

\section{P2YI 2 inhibitors}

The anti-viral combination Lopinavir/Ritonavir causes increased serum concentrations of Ticagrelor through CYP3A4 inhibition and therefore poses an excess bleeding risk. Use of Ticagrelor has been discouraged in the USA and Canada during the pandemic. Clopidogrel, on the other hand, may not provide sufficient platelet inhibition during concomitant administration of Lopinavir/Ritonavir. Use of Prasugrel has been deemed safe. ${ }^{(5)}$

\section{Anti-coagulants}

Heparin is preferred, as its anti-coagulant effect can be monitored. No major interactions have been reported with 


\begin{tabular}{|c|c|c|}
\hline Drug & Dosing & Notes \\
\hline Aspirin & $\begin{array}{l}\text { Loading dose of I } 50 \text { - 300mg orally as soon as possible for all patients without } \\
\text { contraindications, then a maintenance dose of } 75-100 \mathrm{mg} \text { daily }\end{array}$ & \\
\hline Clopidogrel & Loading dose of $600 \mathrm{mg}$ orally, then a maintenance dose of $75 \mathrm{mg}$ daily & \multirow{3}{*}{$\begin{array}{l}\text { A more potent P2Y } 12 \text { inhibitor } \\
\text { (ticagrelor or prasugrel) is favoured. } \\
\text { If these are not available or are } \\
\text { contraindicated, then clopidogrel } \\
\text { should be used. Administration before } \\
\text { (or at latest at the time of) PCl is } \\
\text { recommended and should be } \\
\text { maintained for } 12 \text { months }\end{array}$} \\
\hline Ticagrelor & Loading dose of $180 \mathrm{mg}$ orally, then a maintenance dose of $90 \mathrm{mg} b d$ & \\
\hline Prasugrel & $\begin{array}{l}\text { Loading dose of } 60 \mathrm{mg} \text { orally, then a maintenance dose of } 10 \mathrm{mg} \text { daily } \\
\text { - In patients weighing }<60 \mathrm{~kg} \text {, the maintenance dose is reduced to } 5 \mathrm{mg} \text { daily } \\
\text { - Prasugrel is contra-indicated in patients with previous stroke } \\
\text { - In patients }>/=75 \text { years of age, Prasugrel is not recommended }\end{array}$ & \\
\hline $\begin{array}{l}\text { Unfractionated } \\
\text { Heparin }\end{array}$ & $\begin{array}{l}\text { - } 70 \text { - } 100 \text { IU/kg IV bolus when no GPIIb/llla inhibitors planned } \\
\text { - } 50 \text { - } 70 \text { IU/kg IV bolus with GP IIb/Illa inhibitors }\end{array}$ & \multirow{2}{*}{$\begin{array}{l}\text { Routine use of Heparin is } \\
\text { recommended IC. Use of Enoxaparin } \\
\text { IV is a lla recommendation }\end{array}$} \\
\hline Enoxaparin & $0.5 \mathrm{mg} / \mathrm{kg} \mathrm{IV}$ bolus & \\
\hline
\end{tabular}

investigational drugs for COVID-19 and parenteral anti-coagulants - except that heparin should not be co-administered with Azithromycin.

NOACs (non-vitamin K antagonist oral anticoagulants) such as rivaroxaban and apixaban, which may be used as part of a triple anti-thrombotic regimen in those with AF undergoing $\mathrm{PCl}$, should be avoided in combination with Lopinavir/ Ritonavir, which acts by inhibition of cytochrome P450 3A4 (CYP3A4) and/or P-glycoprotein (P-gP) activity - thereby increasing bleeding risk. ${ }^{(5)}$

\section{Anti-arrhythmics in the cath lab}

Prior to arrival in the cath lab, correction of reversible triggers for arrhythmia such as hypoxia, hypovolaemia, electrolyte abnormalities (hypokalaemia, hypomagnesaemia), metabolic acidosis and excessive use of catecholamines should be addressed. In patients with recurrent VT/VF or uncontrolled atrial fibrillation/atrial flutter, intravenous amiodarone is the anti-arrhythmic of choice. Its use in combination with hydroxychloroquine and/or azithromycin should be avoided due to QT prolongation and a pro-arrhythmic potential, especially Torsades de Pointes VT. Therapy of Torsades $V T$ includes withdrawal of QT-prolonging drugs, maintaining serum potassium $>4.5 \mathrm{mEq} / \mathrm{L}$, IV magnesium supplementation and increasing heart rate (with IV Isoproterenol or temporary pacing). ${ }^{(5)}$

\section{Cardiogenic shock}

Inotropes and/or vasopressors may be required to maintain hemodynamic stability in the cath lab. In critically ill COVID-19 patients at risk for shock, a mixed aetiology of cardiogenic (large Ml, severe myocarditis, LV dysfunction) and septic shock may need to be considered. Selecting the proper agent according to the patient's clinical profile and limiting infusion to the shortest time and lowest dose possible, are all important.

\section{Post ACS statins and RAAS-inhibitors}

Co-administration of Lopinavir/Ritonavir with statins may result in myopathy. Simvastatin is contraindicated due to the risk of rhabdomyolysis. Atorvastatin or Rosuvastatin should be administered at the lowest possible dose. ${ }^{(12)}$

Angiotensin Converting Enzyme Inhibitor (ACEI)/Angiotensin Receptor Blockers (ARB) are often used in the post ACS setting. Although concern has been raised with the finding that these drugs increase levels of ACE-2, it is recommended that their use be continued for hypertension, heart failure and post -MI LV dysfunction. ${ }^{(5)}$

\section{ELECTIVE CARDIOVASCULAR PROCEDURES DURING THE COVID-I 9 PANDEMIC}

The knee-jerk reaction to the pandemic is to cancel all elective procedures until some point when it is considered safe to resume normal hospital activities. The underlying rationale seems solid: These procedures are not urgent and can therefore safely be postponed, patient contact to potentially infected individuals is reduced, healthcare personnel are not exposed to potential COVID-19 carriers, with a reduced infection risk, and hospital resources particularly ICU beds and ventilators are preserved for potentially sick COVID-19 patients.

This may be the correct approach in many specialities, but is not the case for many patients with underlying cardiovascular disease. Although a procedure may not necessarily be immediately indicated to avert a life-threatening insult in the "elective" cohort of patients, deferral of a required intervention may lead to a deterioration of the underlying condition for a significant number of patients in this group. 
TABLE III: Timing of cardiovascular procedures.

\begin{tabular}{l|l|l}
\hline Category & \multicolumn{1}{c}{ Time } & \multicolumn{1}{c}{ Example } \\
\hline Urgent & $<1$ month & Severe symptomatic valve disease (especially AS or MS), non-infarct-related artery PCl post-AMI \\
\hline Essential & $1-3$ months & Subacute valve procedures, transplant workup \\
\hline Elective & $3-6$ months & ASD, PDA, PFO, LAA closure \\
\hline Optional & & Revascularisation of patients with chronic CAD \\
\hline
\end{tabular}

The timing of so-called "elective" procedures in this patient population therefore requires more thoughtful consideration.

\section{Time delay of elective procedures}

The vast majority of therapeutic procedures in patients with CV disease are not optional and cannot be postponed indefinitely. Unfortunately, there are no data to guide us regarding the acceptable delay of a therapeutic intervention in patients with cardiovascular disease. Clinicians will therefore have to use their judgement and knowledge of the natural history of the disease to assess how long a patient could potentially wait to have a procedure. Table III shows a classification of timing for common cardiovascular interventions.

\section{Risk of patient infection during hospital stay}

The virus has to date not been shown to be transferred via long-range airborne transmission. The secondary attack rate for household contacts is $10 \%-15 \% .{ }^{(20,21,22)}$ The risk of transmission otherwise is unknown; in particular: it is not certain what the risk of transfer between 2 masked individuals is who practice good hand hygiene. Overall it seems the risk of a patient contracting the virus in hospital with the necessary precautions would be very low.

\section{Risk to HCP}

The easiest way to mitigate the risk of HCP infection would be to do procedures only on COVID-19 negative patients or patients who have fully recovered from the infection. However, asymptomatic COVID-19 patients are not uncommon (up to $50 \%$ in some studies). ${ }^{(23)}$ In addition, patients who have contracted the virus are already infectious a few days prior to developing symptoms and can thus unwittingly infect those in contact with them. ${ }^{(24)}$

Given the cost and the delay in obtaining test results, performing a routine COVID-19 screening nasal swab on every asymptomatic patient undergoing an elective procedure is not feasible in most healthcare settings in South Africa. Clinical screening looking at a combination of symptoms is possibly helpful, but the overall sensitivity to detect patients with
COVID- 19 is likely to be poor, as patients may be asymptomatic while infective.

Nonetheless the risk of transmission to the HCP for most cardiovascular procedures (i.e. procedures in which there is a low exposure to respiratory secretions and aerosol production) is low, provided basic strategies to prevent infection (surgical masks for both patient and HCPs and good hand hygiene) are employed. ${ }^{(25,26)}$

It would therefore be reasonable to proceed with interventions where the risk of virus exposure is low. This would be the case for most routine procedures performed in the angio suite.

\section{Facility resource utilisation}

As it is likely that the hospital resources need to be diverted to sick COVID- 19 patients, it is important to preserve ICU beds. Procedures on patients who are expected to have a significant peri-procedural ICU stay, should be deferred if possible. Hospital stay should be as short as possible in order to minimise the chance of infection of either patients or HCP.

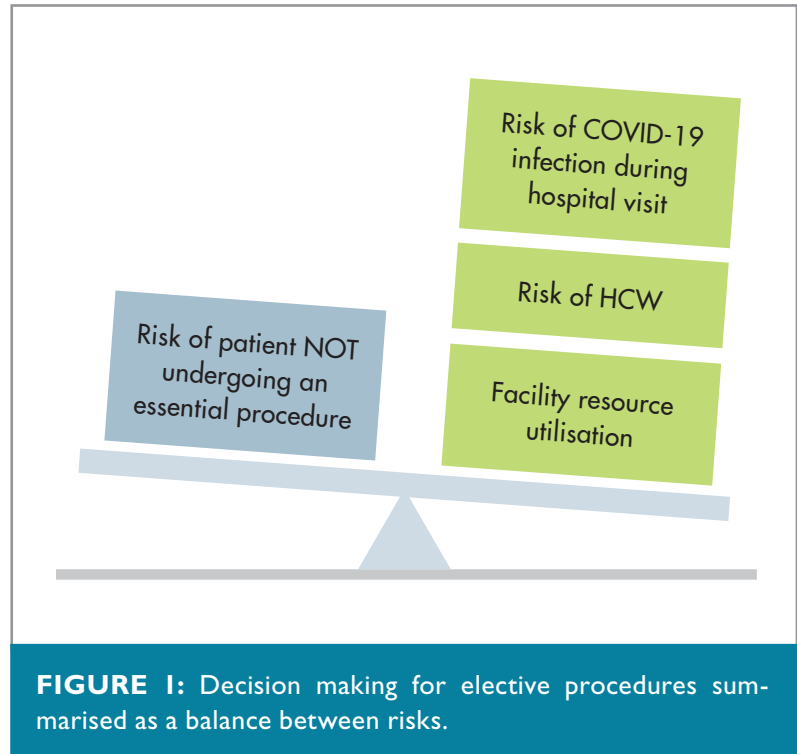


Figure I provides a pictorial summary of some of the issues which need to be considered prior to deciding to proceed to the cath lab, and Figure 2 provides an algorithm to help guide the decisions once the issues have been weighed up.

\section{CONCLUSION}

Projections by the $\mathrm{WHO}$ are that the COVID-19 pandemic will persist into the foreseeable future, until either a vaccine is widely available or there is widespread "herd" immunity. During this period we, as interventional cardiologists in South Africa, will have to become acquainted with a new paradigm that requires us to prepare and plan well beyond the technical interventional details and anatomical complexities of our patient and consider the safety of the entire team of healthcare professionals and others who work in the cath lab. In this perspective review, we as the SASCl executive committee, have attempted to provide a base of core issues to consider as you adapt your practice to the new environment.

Conflict of interest: none declared.

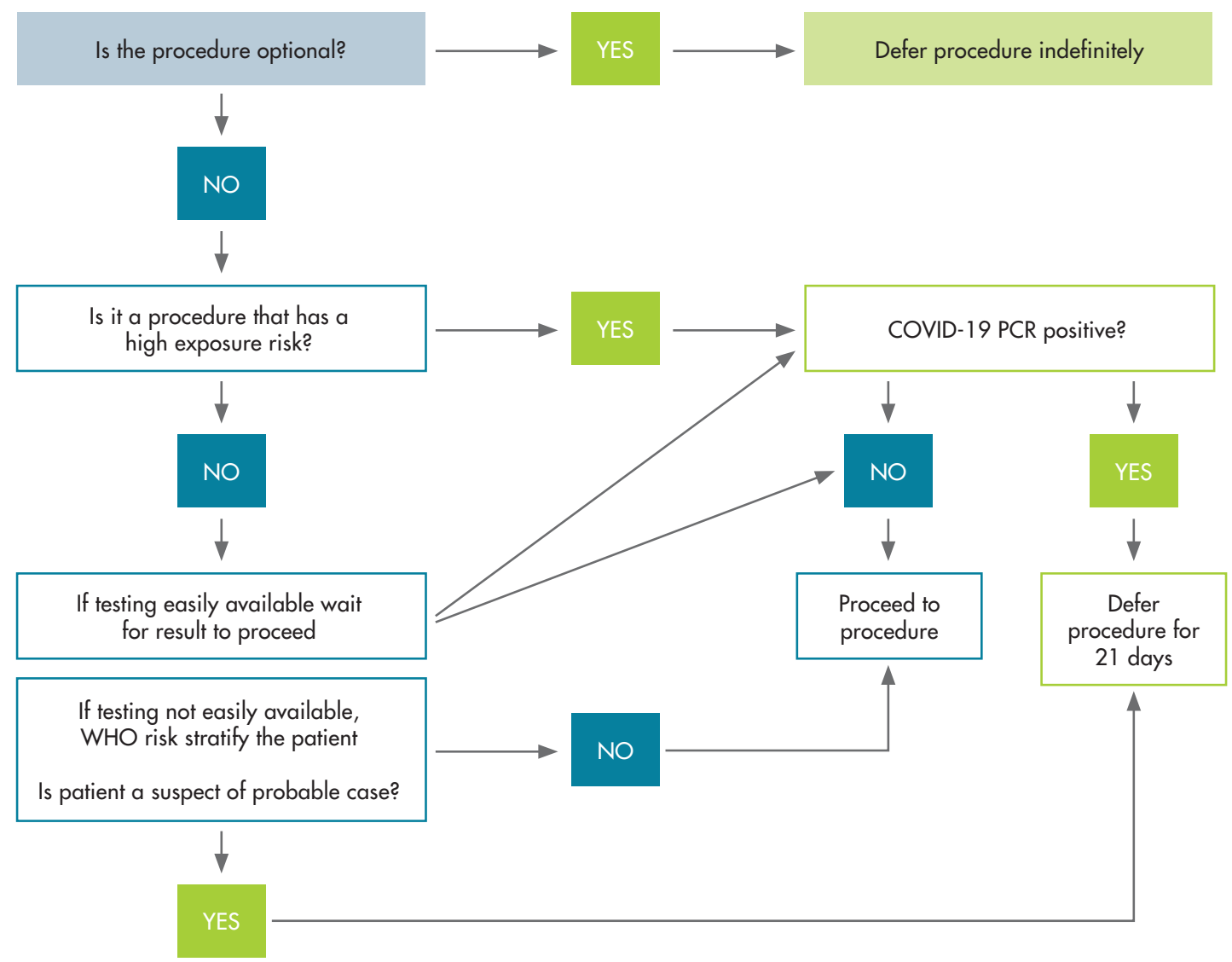

FIGURE 2: Flow diagram to delineate management of patients requiring elective procedures. 


\section{REFERENCES}

I. Burrer SL, de Perio MA, Hughes MM, et al. Characteristics of health care personnel with COVID-19 - United States, February 12 April 9, 2020 MMWR Morb Mortal Wkly Rep (online). 17 Apr 2020 [cited 4 May 2020];69(15):477-48I. Retrieved: http://www.cdc.gov/mmwr/volumes/69/ $\mathrm{wr} / \mathrm{mm} 69 \mid 5 \mathrm{e} 6 . \mathrm{htm}$ ?s_cid=mm69|5e6_w.

2. Chou R, Dana T, Buckley DI, et al. Epidemiology of and risk factors for coronavirus infection in health care workers. Ann Intern Med 20205 May 5.

3. World Health Organization. Global surveillance for COVID-19 caused by human infection with COVID-19 virus: interim guidance. 20 March 2020 (online). Geneva: World Health Organization;2020. Retrieved: https://apps. who.int/iris/handle/I0665/33/506.

4. John T-J, Hassan K, Weich H. Donning and doffing of personal protective equipment (PPE) for angiography during the COVID-19 crisis. European Heart Journal Oxford Academic (online). 2020 [cited I5 May 2020]. Retrieved: https://academic.oup.com/eurheartj/advancearticle/doi/l0.1093/ eurheartj/ehaa283/5819446.

5. Andreini D. ESC guidance for the diagnosis and management of $c \vee$ disease during the COVID-19 pandemic. 2020 Apr.

6. Chieffo A, Stefanini GG, Price S, et al. EAPCI Position Statement on invasive management of acute coronary syndromes during the COVID-19 pandemic. Eur Heart J (online). I4 May 2020;4| (19):| 839- | 85।. Retrieved: https://doi. org/10.1093/eurheartj/ehaa381.

7. De Filippo O, D'Ascenzo F, Angelini F, et al. Reduced rate of hospital admissions for ACS during Covid-19 outbreak in Northern Italy. N Engl Med (online). 28 Apr 2020 [cited 25 May 2020]. Retrieved: http://www.ncbi. nlm.nih.gov/pubmed/32343497.

8. Mahmud E, Dauerman HL, Welt FG, et al. Management of acute myocardial infarction during the COVID-19 pandemic. J Am Coll Cardiol 2020 20 Apr 20.

9. Huang C, Wang Y, Li X, et al. Clinical features of patients infected with 2019 novel coronavirus in Wuhan, China. Lancet. I5 Feb 2020 395( I 0223):497-506

10. Tam CCF, Cheung KS, Lam S, et al. Impact of coronavirus disease 2019 (COVID-19) outbreak on ST-Segment-elevation myocardial infarction care in Hong Kong, China. Circulation. Cardiovascular quality and outcomes. NLM (Medline) 2020; 13

11. Stefanini GG, Montorfano M, Trabattoni D, et al. ST-elevation myocardia infarction in patients with COVID-19: Clinical and angiographic outcomes. Circulation 30 Apr 2020

12. Driggin E, Madhavan MV, Bikdeli B, et al. Cardiovascular considerations for patients, health care workers, and health systems during the COVID-19 pandemic. Journal of the American College of Cardiology 2020;75: 2352-237I.

13. Kwong JC, Schwartz KL, Campitelli MA, et al. Acute myocardial infarction after laboratory-confirmed influenza infection. N Engl J Med 25 Jan 2018:378(4):345-353

14. Bikdeli B, Madhavan MV, Jimenez D, et al. COVID-19 and thrombotic or thromboembolic disease: Implications for prevention, antithrombotic therapy, and follow-up. J Am Coll Cardiol 2020 Apr.

15. Ibanez B, James S, Agewall S, et al. 2017 ESC Guidelines for the management of acute myocardial infarction in patients presenting with ST-segment elevation: The Task Force for the management of acute myocardial infarction in patients presenting with ST-segment elevation of the European Society of Cardiology. Eur Heart J (online). 26 Aug 2017;39(2):119-177. Retrieved: https://doi.org// 0.1093/eurheartj/eh×393.

16. Armstrong PW, Gershlick $\mathrm{AH}$, Goldstein P, et al. Fibrinolysis or primary PCl in ST-segment elevation myocardial infarction. N Engl J Med II Apr 2013 Apr;368(I5):1379-1387.
17. Anderson JL. 2013 ACCF/AHA guideline for the management of STelevation myocardial infarction: A report of the American College of Cardiology Foundation/American Heart Association Task Force on practice guidelines. Circulation; 2013;127.

18. Roffi M. 2015 ESC Guidelines for the management of acute coronary syndromes in patients presenting without persistent ST-segment elevation: Task Force for the Management of Acute Coronary Syndromes in Patients Presenting without Persistent ST-Segment Elevation of the European Society of Cardiology. EHJ 20 I 6;37(3):267-3।5.

19. Shah R. Opiate use in the cath lab. European Heart Journal EHJ (online). 21 Feb 2018 (cited 27 May 2020);39(8):642-645. Retrieved: https:// academic.oup.com/eurheartj/article/39/8/642/4833993.

20. Böhmer MM, Buchholz $U$, Corman VM, et al. Articles investigation of a COVID-19 outbreak in Germany resulting from a single travel-associated primary case: a case series. 2020 (cited 25 May 2020). Retrieved: www. thelancet.com

21. Burke RM, Midgley CM, Dratch A, et al. Active monitoring of persons exposed to patients with confirmed COVID-19 - United States, JanuaryFebruary 2020. MMWR Morb Mortal Wkly Rep 6 Mar 2020;69(9):245-246.

22. Park SY, Kim Y-M, Yi S, et al. Coronavirus disease outbreak in call center South Korea. Emerg Infect Dis 2020 Aug;26(8).

23. Li R, Pei $\mathrm{S}$, Chen B, et al. Substantial undocumented infection facilitates the rapid dissemination of novel coronavirus (SARS-CoV2). Science $16 \mathrm{Mar}$ 2020; 1 6;368(6490).

24. He $X$, Lau EHY, Wu P, et al. Temporal dynamics in viral shedding and transmissibility of COVID-19. Nat Med 2020;26(5)

25. $\mathrm{Ng} \mathrm{K}$, Poon BH, Kiat Puar TH, et al. COVID-19 and the risk to health care workers: A case report. Ann Intern Med 202016 Mar.

26. Wong SCY, Kwong RTS, Wu TC, et al. Risk of nosocomial transmission of coronavirus disease 2019: an experience in a general ward setting in Hong Kong. J Hosp Infect 2020 I Jun. 